\title{
Current paradigms in aortic arch repair: Striking the balance between open surgery and endovascular repair
}

\author{
Prashanth Vallabhajosyula, MD, MS, and Wilson Y. Szeto, MD
}

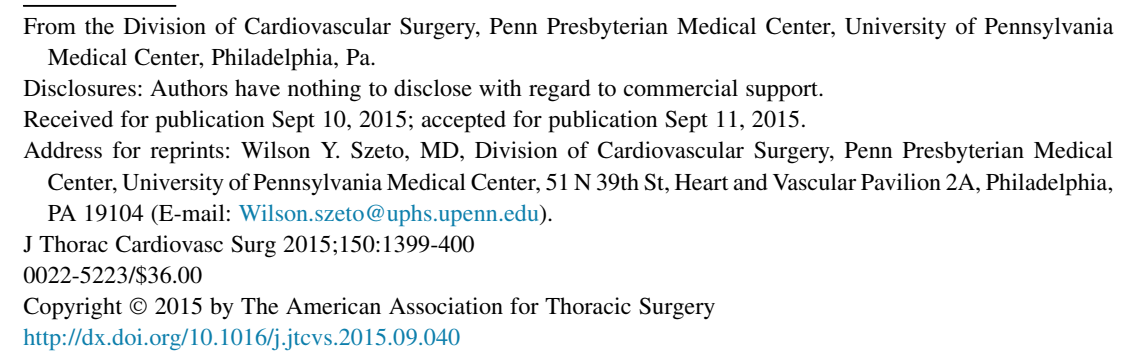

Surgical treatment strategies for aortic arch pathology have traditionally involved open techniques of total arch replacement with reimplantation of the great arteries. However, the ongoing rapid progress in thoracic endovascular aortic repair (TEVAR) technology during the past 15 years is challenging the traditional open repair paradigms. Furthermore, innovative hybrid repair techniques combining open and endovascular repairs have expanded the indications as well as broadened the scope of aortic pathologies amenable to surgical therapies. ${ }^{1}$ The series of articles in this issue of the Journal from various centers advocating for hybrid techniques in treating aortic arch pathologies is a reflection of the rapid adaptation of stent graft technology. As the field evolves, we need to critically review the current status of operative alternatives for treating aortic arch pathologies and also seek a more cohesive approach to studying this complex condition.

Essential to determining the best treatment strategy for arch pathology is the understanding of the aortic arch in conjunction with the anatomy of the ascending and descending thoracic aorta (DTA). Furthermore, the acuity of arch pathology as well as a patient's comorbidity burden must be carefully considered. These factors not only determine the extent of aortic resection and reconstruction required at the index operation, but also whether a given patient can tolerate an extensive single-stage strategy versus a many-staged therapeutic strategy. Traditional open repair remains the gold standard against which all endovascular or hybrid repair strategies must be compared. Despite the definitive nature and durability of the open approach, the morbidity and mortality associated with open repair is not minimal, and many patients are simply not ideal candidates for open surgery that requires the use of cardiopulmonary bypass and circulatory arrest with complex cerebral perfusion strategies. A hybrid approach provides a new tool to modify and to improve our open techniques, and serves as a synergistic rather than competitive therapeutic strategy.

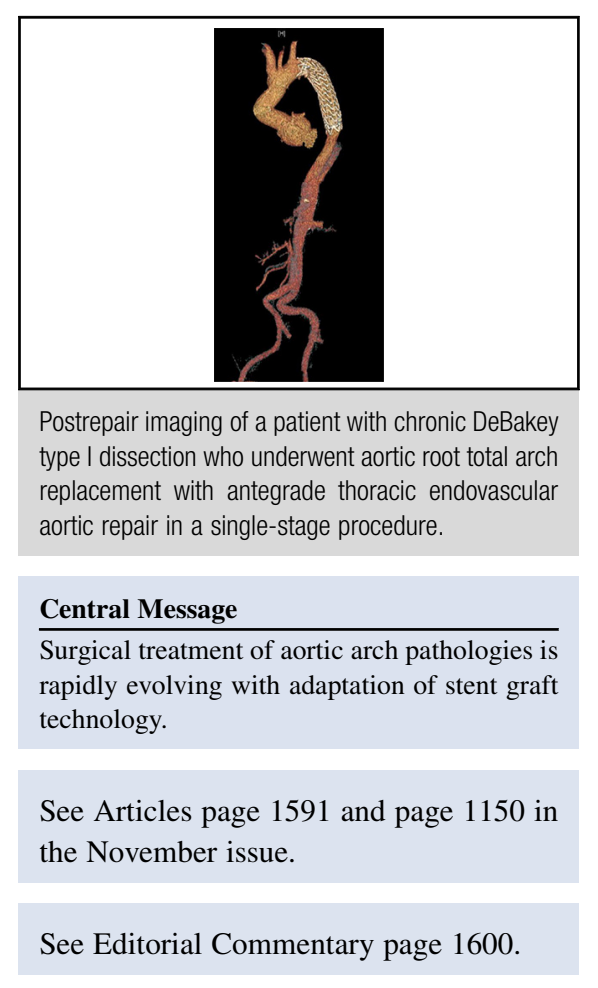

The impetus for hybrid approaches in the treatment of complex aortic arch pathologies is exactly this synergistic effect of endovascular technique with open surgery. Complex arch pathology that includes both the proximal ascending and the distal descending aorta remains a clinical challenge. Extensive single-stage open procedure remains uncommonly performed because most of the patients in need of the procedure are elderly individuals with significant comorbidities who will simply not tolerate this extensive approach. For the staged approach, even advocates agree that a significant number of patients who undergo the first stage do not progress to the second open stage for a variety of reasons, leaving these patients with extensive aortic pathologies that are not completely treated. The use of a hybrid approach facilitates the treatment of extensive aortic pathology by combining the benefits of open proximal reconstruction with the minimally invasive nature of a second staged TEVAR treatment.

This concept of endovascular facilitation of complete aortic therapy is repeatedly seen in the series presented in this issue of the Journal. Creative investigators have designed hybrid approaches as simple as the frozen 
elephant trunk wherein generic TEVAR endografts are used to augment total arch replacement to include treatment of distal aortic pathology. These techniques minimize the duration of hypothermic circulatory arrest during arch reconstruction and have the potential to eliminate or facilitate second-stage distal aortic reconstruction. This concept has been embraced by other investigators and a specific platform designed for this approach has been developed. The Thoraflex hybrid prosthesis (Vascutek, Glasgow, UK) comprises a distal stent graft of variable diameter and length connected to a proximal 3-branch graft. The prosthesis is designed to address arches with DTA pathology in a single-stage operation. During the period of circulatory arrest the stent graft portion of the device is deployed into the DTA, and then the distal anastomosis is performed. Each head vessel is then individually anastomosed to the 3 limbs of the proximal portion of the hybrid stent graft device. The results in Europe have been very encouraging and hybrid stent graft devices are currently being trialed in the United States.

With the recent development of endografts designed for the aortic arch, this is only the beginning. TEVAR designs continue to rapidly develop. Branched and fenestrated endografts are currently under investigation and total endovascular therapy may 1 day be a viable treatment therapy for arch pathology. Furthermore, proximal ascending aortic and aortic valve pathology are currently treated with catheter-based therapies with increasing frequency and success. However, much more work and many detailed investigations are required to understand outcomes associated with endovascular repair of arch pathologies. The same intense scrutiny and research in stroke prevention with open aortic surgery need to be applied to these new therapeutic strategies. Cerebral embolic protection strategies may 1 day be an integral part of these procedures. Finally, patient selection and therapy selection remain to be further defined. A comprehensive multidisciplinary aortic team will be the key to success and the cardiovascular surgeon must be fully engaged.

With the rapid technologic advancements in TEVAR designs, it is critical that cardiovascular surgeons embrace innovation and own this new paradigm of treatment. Technology should not be seen as an obstacle but rather a transformative catalyst to improve surgical technique and outcome. At the same time, we must refrain from overly enthusiastic acceptance of new therapies without evidence-based investigations. Cardiovascular surgeons trained in both open and endovascular surgery are perfectly poised to understand and study the role of open surgery, endovascular therapy, and hybrid procedure in treating aortic arch pathologies. With this balanced philosophy we can develop innovative therapeutic strategies for patients in whom no other surgical options existed in the past.

\section{Reference}

1. Szeto WY, Bavaria JE. Hybrid repair of aortic arch aneurysms: combined open arch reconstruction and endovascular repair. Semin Thorac Cardiovasc Surg. 2009;21:347-54. 